Western University

Scholarship@Western

Mechanical and Materials Engineering

Mechanical and Materials Engineering

Publications

Department

2018

\title{
The Development and Numerical Modelling of a Representative Elemental Volume for Packed Sand
}

Ashraf Thabet

The University of Western Ontario

Anthony G. Straatman

uwo, agstraat@uwo.ca

Follow this and additional works at: https://ir.lib.uwo.ca/mechanicalpub

Part of the Materials Science and Engineering Commons, and the Mechanical Engineering Commons

Citation of this paper:

Thabet, Ashraf and Straatman, Anthony G., "The Development and Numerical Modelling of a Representative Elemental Volume for Packed Sand" (2018). Mechanical and Materials Engineering

Publications. 10.

https://ir.lib.uwo.ca/mechanicalpub/10 


\title{
The development and numerical modelling of a Representative Elemental Volume for packed sand
}

\author{
Ashraf Thabet, Anthony G. Straatman* \\ Department of Mechanical \& Materials Engineering, Western University, London N6A 5B9, Canada
}

\section{H I G H L I G H T S}

- Idealized geometric model introduced to characterize packed sand in pore-level computations.

- Geometric model has spherical particles packed randomly, but with spatial periodicity.

- Comparisons show that idealized model gives predictions similar to actual sand REV.

- Particle size variation shown to not be important in computational model.

- Influence of temperature on properties shown to be important in interstitial exchange.

\section{A R T I C L E I N F O}

\section{Article history:}

Received 6 February 2018

Received in revised form 21 April 2018

Accepted 23 April 2018

Available online 30 April 2018

\section{Keywords:}

Computational modelling

Packed bed

Porous media

Convective heat transfer

\begin{abstract}
A B S T R A C T
This article describes a comparison between a packed-sand geometric model derived from experimental digitization of a small volume of sand and from a mathematical model (Yet Another Development Engine, YADE) that produces a random packing of spherical particles. As the application of the model is towards smoldering combustion, the comparison focuses on the pressure drop and convective heat transfer at very low Reynolds numbers and a wide range of high temperatures. The comparison shows that the YADE geometric model gives results that are in good agreement with the digitized packed-sand model in the range of Reynolds numbers considered, which, given the cost and time saving of using a mathematical model, is an extremely useful result. The geometric model was also used to explore the influence of particle size variation and temperature dependence of air properties affects the flow and convective exchange at temperatures relevant to smouldering combustion. A correlation is presented to demonstrate how the heat transfer results can be put into a compact form for use in computations using a porous continuum approach.
\end{abstract}

(c) 2018 Elsevier Ltd. All rights reserved.

\section{Introduction}

Significant progress has been made with respect to understanding the behavior of fluid flow, heat and mass transfer, and chemical reaction in permeable porous media. Fluid flow and heat transfer in porous materials is prevalent in numerous applications in science and engineering. For example, in chemical engineering, transport in porous media is related to filtering and drying applications, packed bed reactors, catalytic converters and fuel cells. In mechanical engineering, applications of porous materials include enhanced heat transfer, evaporative cooling, insulation and heat sinks. In environmental applications, porous media provide context for the study of groundwater flows and contaminant transport in groundwater.

\footnotetext{
* Corresponding author.

E-mail address: astraatman@eng.uwo.ca (A.G. Straatman).
}

Another important environmental application of porous media is in smoldering combustion phenomenon, which is characterized as an oxygen-limited flameless form of combustion with a slow propagation rate and a relatively low temperature (in the context of combustion). The phenomenon is extremely complex and includes heterogeneous chemical reactions and transport of heat, mass, and momentum of multiple phases (Rein, 2009). Motivation for the study of smoldering combustion has arisen primarily from the possible economic and pollution-related impact of this phenomenon. In the USA, it has been reported that an estimated 31,200 smoldering fires that occurred during 2001 caused property losses of about US\$400 million (Rein, 2009). In Canada, since 1990, "wildland fires" have consumed an average of 2.5 million hectares a year, especially during prolonged periods of drought (NRC). Such fires can smolder underground all winter and then re-emerge at the surface in the spring. In this respect, the potential for significant economic damage and losses provides motivation for better 


\section{Nomenclature}

\begin{tabular}{|c|c|}
\hline$a_{f s}$ & specific interfacial surface area of porous media, $\mathrm{m}^{2} / \mathrm{m}^{3}$ \\
\hline$A_{f s}$ & interfacial surface area of porous media, $\mathrm{m}^{2}$ \\
\hline$C_{p}$ & specific heat capacity, $\mathrm{J} \mathrm{kg}^{-1} \mathrm{~K}^{-1}$ \\
\hline$d$ & particle diameter, $\mathrm{m}$ \\
\hline$L$ & length scale of porous medium or REV length, m \\
\hline$g$ & acceleration of gravity, $\mathrm{m} \mathrm{s}^{-2}$ \\
\hline$h_{f s}$ & $\begin{array}{l}\text { interstitial heat transfer coefficient between the solid } \\
\text { and gas phases, } \mathrm{W} \mathrm{m}^{-2} \mathrm{~K}^{-1}\end{array}$ \\
\hline$k$ & thermal conductivity, $\mathrm{W} \mathrm{m}^{-1} \mathrm{~K}^{-1}$ \\
\hline$k_{f e}$ & effective conductivity of fluid phase, $\mathrm{W} \mathrm{m}{ }^{-1} \mathrm{~K}^{-1}$ \\
\hline$k_{s e}$ & effective conductivity of solid phase, $\mathrm{W} \mathrm{m}{ }^{-1} \mathrm{~K}^{-1}$ \\
\hline K & permeability of the medium, $\mathrm{m}^{2}$ \\
\hline
\end{tabular}

$C_{E} \quad$ form drag coefficient for porous medium

$P \quad$ pressure, $\mathrm{Pa}$

$R \quad$ specific gas constant of air, $\mathrm{J} \mathrm{kg}^{-1} \mathrm{~K}^{-1}$

$\mathrm{V} \quad$ Darcy velocity, $\mathrm{m} \mathrm{s}^{-1}$

$\mathrm{u} \quad$ fluid velocity vector, $\mathrm{m} \mathrm{s}^{-1}$

$\dot{m} \quad$ mass flow rate, $\mathrm{kg} / \mathrm{s}$

$T_{f} \quad$ temperature of fluid constituent of porous media

$T_{s} \quad$ temperature of solid constituent of porous media

$\mathrm{Pr} \quad$ Prandtl number

$\rho \quad$ density, $\mathrm{kg} \mathrm{m}^{-3}$

$\mu \quad$ dynamic viscosity, Pa s understanding the smoldering process and development of preventative strategies, which can minimize its harmful effects on climate, the environment and human and animal life.

A second motivation for better understanding smoldering combustion is to explore the feasibility of its use as a remediation technique. To this end, a recent application called Self-sustaining Treatment for Active Remediation (STAR) has been developed based on in-situ controlled smoldering combustion of contaminated soils or sands. The source of fuel for this technology is the contaminant in the porous soil or sand, which is ignited and then slowly oxidized using hot air, which is delivered through a well to the target contaminated zone. Following ignition - and provided a sufficient amount of ambient or combustion air is supplied - the process is self-sustaining, without the need for external injected fuel (Savron Smoldering Solutions).

Critical to developing an understanding of smoldering combustion phenomenon is knowledge and characterization of heat and mass transfer (at combustion-level temperatures) in packed sands and soils, and these processes can be investigated both experimentally and computationally. Experimental studies can provide the insight into the fundamental processes, which can then be modelled mathematically and included in computer codes that can be used to conduct parametric studies. Numerical modelling can be done at both the pore-level and the porous-continuum (volume-averaged) level. In this manner, detailed information can be established by conducting highlyresolved simulations on representative volumes of porous media, which can then be used to develop parameters required to mathematically close the volume-averaged transport equations, which can be used to study large porous domains. A critical element required to conduct useful and accurate pore-level simulations is a geometric model that correctly mimics the porous structure in which the phenomenon occurs. Such a geometric model is called a Representative Elemental Volume (REV), which is defined as a small volume that is representative of the larger domain (which can be comprised of millions or billions of particles). Thus, one goal of the present article is to address the specific task of identifying a suitable REV for packed sand that can be used to conduct simulations to understand the pore-level activity associated with flow, convection, reaction and mass transfer at conditions associated with smoldering combustion.

In general, permeable porous media are heterogeneous materials comprised of a solid microstructure (or matrix) and a fluid that fills the space unoccupied by the matrix. The structure and material of the porous media play an important role in the physical behavior of the media and in the interaction of the matrix with the fluid phase (Khan et al., 2015). The matrix can take a wide variety of forms, including networks of interconnected ligaments, interconnected spherical voids and packed beds of particles. The specific type of porous structure affects the amount of internal interfacial area between the solid and fluid phases (interstitial area) and the way in which the fluid flows through the medium. A packed bed is a heterogeneous system composed of solid particles and a fluid flowing in the interstitial space between the particles. Packed beds represent a particular type of permeable porous structure and its geometric properties are affected by particle size, particle size variation and particle shape.

Studies on fluid flow and convective heat transfer in packed particle beds has received extensive attention in the last century, particularly during the 1970s. Empirically-derived correlations show that the heat transfer coefficient is dependent on the porosity, particle size, and shape of the packed bed as well as on the Prandtl number of the fluid and the Reynolds number derived from the range of the particle diameters (Vafai, 2015). Dimensionless analysis of the problem suggests that a general correlation for the interstitial Nusselt number of the form (Wakao and Kaguei, 1982):

$N u_{d}=\frac{h_{s f} d}{k_{f}}=\mathrm{a}+\mathrm{c} \operatorname{Pr}^{m} \operatorname{Re}_{d}^{n}$

can be used to capture the convective exchange, where $h_{s f}$ is the interstitial convective exchange coefficient, $d$ is the particle diameter and $k_{f}$ is the fluid conductivity. $a, c, m$ and $n$ are unknowns that require experiments or pore-level computation to determine. Wakao et al. (1979) published their well-known correlation for packed beds of spheres:

$N u_{d}=2+1.1 \operatorname{Pr}^{1 / 3} \operatorname{Re}_{d}^{0.6}$

which is valid over the range $0.7<\operatorname{Pr}<1$ and $15<\operatorname{Re}<8500$, and was established from packed beds of particles with diameters in the range 10-130 mm (Wakao and Kaguei, 1982; Wakao et al., 1979). This correlation has been widely used in studies of packed beds reported in the literature and has been extended to include consideration of exchange at lower Reynolds numbers and of particles with small diameters and geometric shapes other than spheres. Kar and Dybbs (1982) proposed:

$N u_{d}=0.004\left(\frac{d_{v}}{d}\right)^{0.35} \operatorname{Pr}^{1 / 3} \operatorname{Re}_{d}^{1.35}$

where $d_{v}=4 \varepsilon / a_{s f}$ is the average void diameter, and $d$ is the particle diameter. This correlation is valid for $R e<75$, but it underestimates the interfacial heat transfer at low Reynolds numbers. Another option is for the second term $\left(\operatorname{Pr}^{m} R e_{d}^{n}\right)$ of the equation to be multiplied by the porosity $(\varepsilon)$. For spherical particles whose porosity is within the range of $0.7<\varepsilon<0.95$ this leads to (Nakayama et al., 2009): 
$N u_{d}=0.07\left(\frac{\varepsilon}{1-\varepsilon}\right)^{2 / 3}\left(\operatorname{Re}_{d} \operatorname{Pr}\right)$

Less information exists in the literature for Reynolds numbers below 30 and Prandtl and Schmidt numbers between 1 and 120 . For Reynolds number less than 20, a recent study by Zanoni et al. (2017) based on combined experimental and computational analysis deduced the convective heat transfer for small sized particles $(0.125-2 \mathrm{~mm})$. This study describes experiments on a column of heated sand wherein temperature measurements were made along the central axis of the column. The interstitial heat transfer coefficient was backed-out of the calculations by systematically adjusting it until the temperature predictions matched those measured in the column (Zanoni et al., 2017). The proposed correlation, valid for Reynolds numbers less than 31 is given as:

$N u_{d}=0.001 \operatorname{Re}_{d}^{1.97} \operatorname{Pr}^{1 / 3}$

It is worth noting that this correlation produces interstitial exchange coefficients that are substantially lower than those extrapolated from Wakao et al. (1979) in the low- $R e_{d}$ range.

Pore-level computations of flow and heat transfer in various types of porous media have also been reported in the literature. Yu et al. (2006) proposed a simple unit-cube geometry model for spherical void phase porous materials (like foams) to characterize the internal structure of graphitic foam (Yu et al., 2006). Karimian and Straatman (2008) used this model for pore-level simulations of convection, but found that the predicted pressure drops were far too low unless blockage factors were introduced. Significant improvement to the unit-cube model was introduced in different studies; for example, Chueh et al. (2014) introduced a 3D REV by which, the thermal conductivity was calculated although the model was periodic in two directions. Dyck and Straatman (2015) created a REV of variable-sized spheres are randomly arranged to produce a foam structure with a specified pore-size and porosity incorporating YADE (Yet Another Development Engine). This model yields far superior results in terms of both pressure drop and heat transfer compared to prior work (Dyck and Straatman, 2015). In terms of packed beds of particles, a study of a simple cubic packing has been used to build a channel of large number of particles (Yang et al., 2010, 2012) and used to study heat transfer and pressure drop. Yang et al. (2010) conducted different experiments, studied the forced convective heat transfer to generate a heat transfer correlation, and finally compared their results to other studies and found that the Wakao et al. (1979) expression may over-predict the Nusselt number. Another study undertaken by Soulaine simulated the flow and heat transfer in a saturated REV micromodel, which was obtained from a 3D micro-CT image following the protocol described in Buchgraber et al. (2012). In this study, complete solution fields normalized by the highest values helped identify some of the important physical properties. More recently, researchers have increasingly turned their attention to the idea of building beds of randomly-packed particles using mathematical models that are based on different shapes and assumptions.

The main purpose of this article is to compare flow and heat transfer from a REV model derived experimentally from segmented images of a small volume of packed sand to a second model derived using the mathematical approach (YADE) described by Dyck and Straatman (2015). Flow and heat transfer results are generated using computational fluid dynamics (CFD) and comparisons are based on overall pressure drop and convective heat transfer. The YADE geometric model is also used to conduct a parametric study to investigate the impact of particle size variation and air property dependence on temperature. A correlation for the interstitial heat transfer is presented which takes into account the temperature influence on the properties and the Reynolds number based on particle diameter. This correlation is presented as a useful and compact means for introducing the interstitial convective heat transfer coefficient in porous continuum calculations that consider local thermal non-equilibrium.

\section{Model formulation}

As the focus of this work is on pore-level simulations of flow and heat transfer in packed sand beds, it is the differential transport equations for mass, momentum and energy that are solved inside the fluid portion of the domain, and this is accomplished using a commercial CFD software. The transport equations for a laminar, incompressible, Newtonian fluid are given as (Vafai, 2015; Kaviany, 1995):

$\frac{\partial \rho_{f}}{\partial t}+\nabla\left(\rho_{f} \mathbf{u}\right)=0$

$\frac{\partial \rho_{f} \mathbf{u}}{\partial t}+\nabla\left(\rho_{f} \mathbf{u u}\right)=-\nabla \mathbf{p}+\nabla\left(\mu_{f} \nabla \mathbf{u}\right)$

$\frac{\partial \rho_{f} c_{p . f} T_{f}}{\partial t}+\nabla\left(\rho_{f} c_{p . f} \mathrm{u} T_{f}\right)=\nabla\left(k_{f} \nabla T_{f}\right)$

where $\mathrm{u}$ is the velocity vector, $p$ is pressure, $T_{f}$ is the fluid temperature, and $\rho_{f}, \mu_{f}, C_{p, f}, k_{f}$, are the fluid density, dynamic viscosity, specific heat and thermal conductivity, respectively, and can either be assumed constant or functions of the local pressure and temperature. Eqs. (6)-(8) are solved inside the fluid domain along with appropriate boundary conditions (described in a subsequent section) using the commercial software ANSYS-CFX ${ }^{\mathrm{TM}}$ or ANSYSFLUENT $^{\mathrm{TM}}$ to compute the pore-level flow and temperature fields.

To draw attention to the parameters that are required to characterize the porous media as a porous continuum, we also introduce the volume-averaged versions of Eqs. (6)-(8) with the addition of a solid-phase energy equation to account for local thermal non-equilibrium (Nield and Bejan, 2017; Whitaker, 1997):

$\frac{\partial}{\partial t}\left(\varepsilon\left\langle\rho_{f}\right\rangle^{f}\right)+\nabla\left(\left\langle\rho_{f}\right\rangle^{f}\langle\mathbf{u}\rangle\right)=0$

$$
\begin{aligned}
\frac{\partial\left\langle\rho_{f}\right\rangle^{f}\langle\mathbf{u}\rangle}{\partial t}+\frac{1}{\varepsilon} \nabla\left(\left\langle\rho_{f}\right\rangle^{f}\langle\mathbf{u}\rangle\langle\mathbf{u}\rangle\right)= & -\varepsilon \nabla\langle p\rangle^{f}+\nabla(\mu \nabla\langle\mathbf{u}\rangle) \\
& -\varepsilon \frac{\mu}{K}\langle\mathbf{u}\rangle-\varepsilon \frac{\left\langle\rho_{f}\right\rangle^{f} C_{E}}{\sqrt{K}}\langle\mathbf{u}\rangle|\langle\mathbf{u}\rangle|
\end{aligned}
$$

$$
\begin{aligned}
& {\left[\varepsilon \frac{\partial \rho_{f} C_{p . f}\left\langle T_{f}\right\rangle^{f}}{\partial t}+\nabla\left(\rho_{f} C_{p . f}\langle u\rangle\left\langle T_{f}\right\rangle^{f}\right)\right]} \\
& \quad=\nabla\left(k_{f e} \nabla\left\langle T_{f}\right\rangle^{f}\right)+h_{f s} a_{f s}\left(\left\langle T_{s}\right\rangle^{s}-\left\langle T_{f}\right\rangle^{f}\right) \\
& (1-\varepsilon) \frac{\left.\partial \rho_{s} C_{p, s} T_{s}\right\rangle^{s}}{\partial t}=\nabla\left(k_{s e} \nabla\left\langle T_{s}\right\rangle^{s}\right)-h_{f s} a_{f s}\left(\left\langle T_{s}\right\rangle^{s}-\left\langle T_{f}\right\rangle^{f}\right)
\end{aligned}
$$

Here, $\varepsilon$ is the porosity defined as the fluid volume divided by the total volume, indicates the volume-average of a quantity over the entire averaging volume (no superscript) or over the fluid $(f)$ or solid $(s)$ portion inside the averaging volume. The additional parameters are the permeability $K\left(\right.$ in $\mathrm{m}^{2}$ ), the Forchheimer drag coefficient $c_{E}$, the interstitial convective exchange coefficient $h_{s f}$ (in $\mathrm{W} / \mathrm{m}^{2} \mathrm{~K}$ ) and the interstitial area per unit volume $a_{s f}$ (in $\mathrm{m}^{2}$ / $\mathrm{m}^{3}$ ). The only other notable feature of the volume-averaged equations is that the fluid and solid conductivities are given as effective conductivities, which means they include dispersion and tortuosity effects in addition to the molecular conductivity itself, i.e.

$k_{e}=k_{\text {cond }}+k_{\text {disp }}+k_{\text {tor }}$ 
for either the fluid $f$ or the solid $s$. The last two terms on the righthand side of Eq. (10) (momentum) are the Darcy and Forchheimer terms, respectively, and represent the viscous and form drag resulting from the interaction between the solid and fluid-constituents in the porous media. Results of the pore-level computations of pressure are area-averaged over the entrance and exit of the REV and then curve-fitted to the equation:

$\frac{\Delta P}{L}=-\frac{\mu}{K}\langle\mathbf{u}\rangle-\frac{\left.\rho_{f}\right\rangle^{f} c_{E}}{\sqrt{K}}\langle\mathbf{u}\rangle|\langle\mathbf{u}\rangle|$

over a range of flow velocities to give appropriate values for the closure coefficients $K$ and $c_{E}$ that can be used in Eq. (10). In cases where the flow in the fluid-saturated porous domain is at a very low velocity and Reynolds number $\left(R e_{d}=\rho_{f} u d / \mu_{f}<1\right)$, the modified Darcy flow model can be used, which reduces Eq. (14) to:

$\frac{\Delta P}{L}=-\frac{\mu}{K}\langle\mathbf{u}\rangle$

The ideal gas equation is incorporated to govern the relation between the thermo-physical properties of air within the domain:

$\rho_{f}=\frac{p}{R T_{f}}$

where $R$ is the gas constant for air.

\section{Geometric modelling of packed sand}

Since a geometric model for an entire packed bed is not reasonable, consideration of a pore-level geometric model focuses on a Representative Elemental Volume or REV. To this end, two different approaches are considered; a model developed from segmented images of a small sample of sand, and an idealized model generated using the YADE model developed by Dyck and Straatman (2015). In both cases, the resulting geometric model is used to development a computational mesh such that CFD can be used to investigate and compare the flow and heat transfer.

\subsection{Segmented image model}

Imaging of a packed sand sample was completed at the Argonne National Lab, Advanced Photon Source Synchrotron, 13-BM-D beamline by the GSECARS research group c (Molnar et al., 2015). The imaging reconstruction procedure and equipment used are detailed in Molnar (2015) and only described briefly herein. In this technique, collected projections were reconstructed into threedimensional data sets using GSECARS specific reconstruction software (Rivers et al., 2010). The input is a 3D dataset of X-ray images averaged over $9.87 \times 9.87 \times 9.87 \mu \mathrm{m}$ in each direction, where the voxel resolution is $9.87 \mu \mathrm{m} /$ voxel (Molnar, 2015; Molnar et al., 2015 ). The output is a REV of $350 \times 350 \times 450$ voxels precisely cropped so that the final dimensions of the selected image is $3.45 \times 3.45 \times 4.44 \mathrm{~mm}$ as shown in Molnar (2015). The sample considered is a uniform quartz with porosity 0.33 and grain size 420-600 $\mu \mathrm{m}$, wherein air saturates the void space.

The images provided by Molnar (2015) and Molnar et al. (2015) required further processing to be used in the present study. After scanning, cutting, and image acquisition were performed (complete explanation of building the sample volume is presented in Molnar (2015), the acquired Raw file of the images was then converted into a STL (Stereo-Lithography) file by ImageJ (An open platform for scientific image analysis) (http://imagej.net/) and an ImageJ plugin called BoneJ (Plugin for image analysis for ImageJ) (http://bonej.org/). ImageJ was applied for binarizing the grayscale values of the images by filtering and thresholding processes. BoneJ was used to compute an iso-surface from the binarized images and to write the STL file. The STL file contains a surface representation of the sand particles as shown in Fig. 1. A consistency check of the STL file was performed in MeshLab (http://meshlab.sourceforge.net/), after which the mesh surface of the sand particles was smoothed and decimated. Finally, the geometry was created by importing the STL file into ANSYS-ICEM ${ }^{\mathrm{TM}}$ (ANSYS ICEM CFD). The fluid continuum that surrounds the sand was identified by subtracting the sand volume from a cube that surrounds the particles region. A tetrahedral mesh was then implemented to fill the fluid space inside the REV. Refinement near the sand surface ensured the quality of the continuous mesh, which is comprised of approximately 6 million cells.

\subsection{Mathematical YADE model}

An analogous pore-level model was generated using the approach described by Dyck and Straatman (2015), except using a modified contact law (Cundall and Strack, 1979) that was developed for spherical particles. In this approach, spheres (primitives) are placed inside a three-dimensional box, which is then compressed allowing the spheres to collide and shift based on the contact law (Cundall and Strack, 1979) until the final porosity is achieved. In order to avoid generating poor quality meshes at contact points between particles, slight deformation (less than $1 \%$ of $d$ ) was allowed to generate better topology of the meshing surface (Yang et al., 2010). This approach produces three-dimensional domains of randomly packed spheres, with the added advantage of spatial periodicity in the $x, y$ and $z$ directions. The procedure of generating the file of 3D randomly-distributed spheres' locations is explained in detail in Dyck and Straatman (2015). By means of written macro, CAD models of the REVs were then created using Solidworks ${ }^{\mathrm{TM}}$ (Dassault Systemes) and then subsequently meshed using the ANSYS Meshing ${ }^{\mathrm{TM}}$ tool (ANSYS Tool). Generation of the mathematical REV can take between a few minutes to a few hours, depending on the number of primitives specified. The minimum number of primitives is that which produces a REV and this is determined by examining the sensitivity of the solution to the number of primitives. A sample YADE model comprised of 50 spherical primitives is given in Fig. 2, which shows both the particles (a) and the fluid space between the particles (b).

\subsection{Computational modelling}

The computational domain was comprised of the REV itself and a short outlet section in the flow direction to eliminate boundary condition issues associated with flow recirculation at the outlet

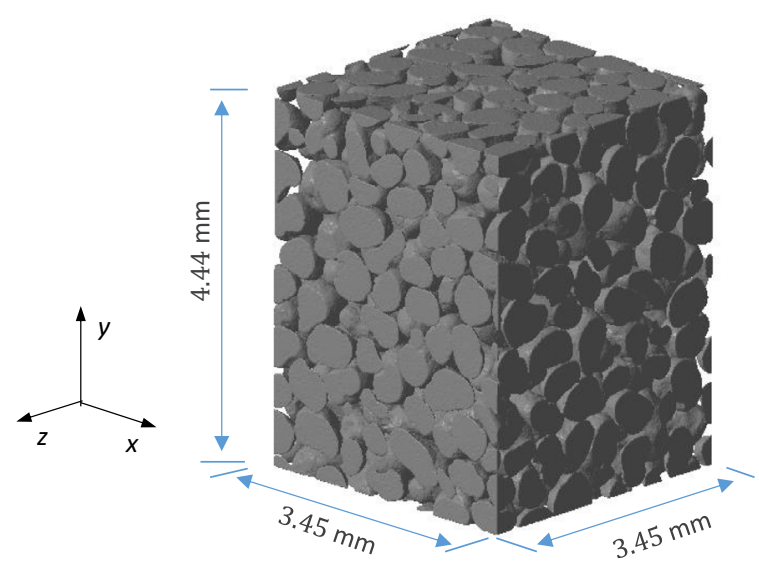

Fig. 1. Three-dimensional geometric model produced from segmented images of a small sample of packed sand (Molnar, 2015). 

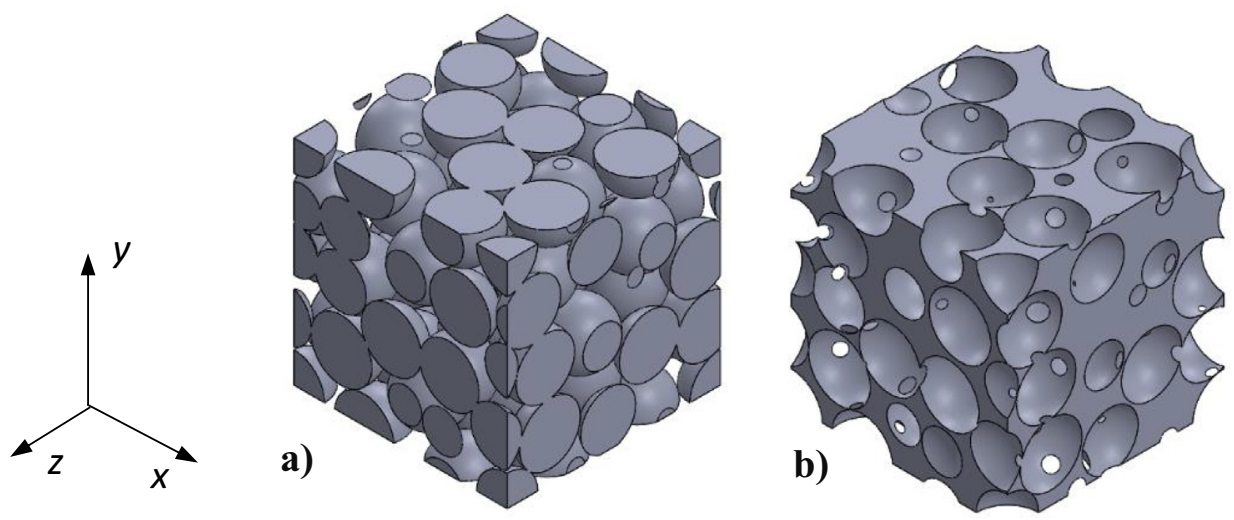

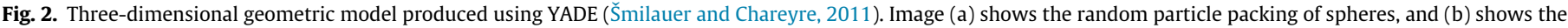
fluid region that requires discretization for analysis. This model is comprised of 50 particles of $d=1.59 \mathrm{~mm}$ and $\varepsilon=0.37$.

of the porous region. Since the geometric models were tested for their sensitivity to the REV size, the addition of the outlet sections presented no inaccuracy in terms of evaluation of the closure parameters. Fig. 3 shows a small section of a computational domain to give an illustration of the grid distribution and the connection and relative length of the outlet section of the domain. For a case where flow specification is in the positive $x$-direction, and referring to Figs. 2 and 3, periodic boundary conditions were imposed on the pairs of $x-y$ and $x-z$ faces of the REV, with a mass flow rate imposed at the inlet $y-z$ face and an outflow boundary condition at the exit $y-z$ face (of the short added section). Noslip, zero-penetration conditions were imposed on the internal surfaces of the REV along with specification of a constant temperature. The choice to conduct simulations with constant particle surface (wall) temperature was made on the basis that it is a comparison of geometric models that is main interest; consideration of a variable wall temperature would require that both the fluid and solid domains would have to be discretized and solved adding much more time to the simulations. The interested reader can consult the Solver Theory Guide for the details of boundary condition implementation either for Fluent ${ }^{\mathrm{TM}}$ (ANSYS Guide) or CFX' (ANSYS CFX).

High-order advection (either QUICK or high-resolution) was utilized for all the transport equations, and pressure-velocity coupling was achieved by employing the SIMPLEC algorithm. The convergence of the simulations was enhanced by slightly modifying the default under-relaxation factors when using Fluent ${ }^{\mathrm{TM}}$ (ANSYS Fluent). Air was specified as the working fluid with either constant or variable thermophysical properties. When variable thermophysical properties were used, the properties of air were set to vary with respect to the local temperature using 6th-degree polynomial functions for conduction, density, viscosity and specific heat. The steady-state problem was simulated until the scaled residuals of

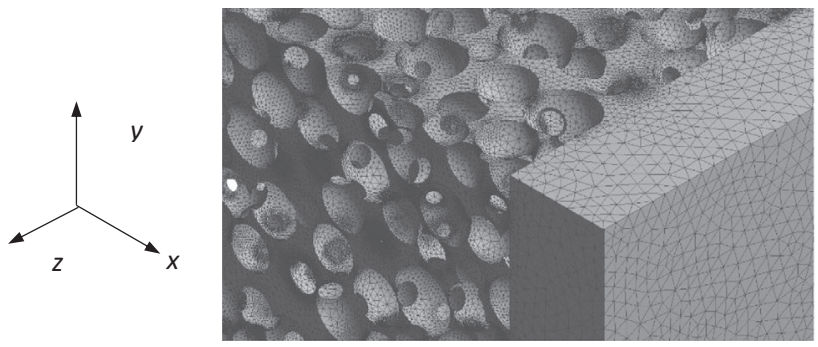

Fig. 3. Image showing computational mesh over a small portion of the REV domain and the exit region. The mesh was generated using the ANSYS meshing ${ }^{\mathrm{TM}}$ Tool. all the transport equations converged to the root mean square below $10^{-4}$. Both CFX and FLUENT produced solutions on the same domains with virtually the same computational time and output results.

\subsection{Processing of computational results}

Referring to Eqs. (10)-(12), the closure coefficients that are sought to characterize the porous media as a porous continuum are the permeability $K$, the Forchheimer drag coefficient $c_{E}$, and the interstitial convective exchange coefficient $h_{s f}$. The interstitial area per unit volume $a_{s f}$ is a geometric parameter and is obtained from the geometric model. The results of the pore-level simulations produced by solving Eqs. (6)-(8) on the pore-level geometric models are processed to obtain the remaining closure parameters.

Closure of the Darcy and Forchheimer drag terms appearing in Eq. (10) is achieved by considering the pore-level hydrodynamics of the REV over the range $0.1 \leq R e_{d} \leq 20$ (suitable for forced convection in packed sand) and a substantial temperature range. Note that because of the low $R e_{d}$ range considered in this work, we neglect the Forchheimer term and simply assume linear behavior to calculate $K$. Since the REVs generated from YADE are spatially periodic in all principle directions, three complete calculations are done on each REV; one for an imposed flow in the $x$ direction, one for an imposed flow in the $y$-direction, and one for an imposed flow in the $z$-direction (with the short outlet section moved accordingly). In this manner, three sets of results are available for each REV produced. The pressure drop is calculated for each flow direction and then averaged as:

$\frac{\Delta P}{\Delta x}=-\frac{1}{3} \sum_{x, y, z} \frac{P_{\text {in }}-P_{\text {out }}}{x_{\text {in }}-x_{\text {out }}}$

where $P_{\text {in }}$ and $P_{\text {out }}$ are area-averaged pressures calculated at the inlet and outlet, respectively, of the computational domain and the denominator is the length of the REV. The permeability $K$ was then determined by fitting a line to all of the $\mu \Delta P / u \Delta x$ data over the range of $R e_{d}$ considered.

Closure of the non-equilibrium energy transport equations requires determination of the interfacial heat transfer coefficient $h_{f s}$. Under fully-developed conditions and constant surface temperature, and neglecting the heat transfer by conduction in the axial direction, the interstitial convection coefficient is established by considering convective heating of a fluid through an isothermal domain:

$\frac{T_{w}-T_{m o}}{T_{w}-T_{m i}}=\exp \left(-\frac{h_{s f} A}{\dot{m} C_{P}}\right)$, 
where $T_{\mathrm{w}}$ is the wall (particle surface) temperature, $T_{m o}$ is the bulk outlet temperature of air, $T_{m i}$ is the bulk inlet temperature of air, $A$ is the interstitial area (in $\left.\mathrm{m}^{2}\right), \dot{m}$ in the mass flow rate of air (in $\mathrm{kg} / \mathrm{s}$ ) and $C_{P}$ is the specific heat of air (in $\mathrm{J} / \mathrm{kg} \mathrm{K}$ ). Solving Eq. (18) for the interstitial heat transfer coefficient gives:

$h_{f s}=-\frac{1}{3} \sum_{x, y, z} \frac{\dot{m} c_{P}}{A_{f s}} \ln \left(\frac{T_{w}-T_{\text {out }}}{T_{w}-T_{\text {in }}}\right)$

where the summation over $x, y, z$ indicates that the results for heating are obtained from three sets of computations on the same domain and then averaged to obtain the final coefficient for a given REV.

\subsection{Sensitivity studies}

Before reporting on comparisons between the segmented image model and the mathematical YADE model, it is prudent to report on the sensitivity study used to determine the influence of the flow and heat transfer results on the grid resolution, the number of primitives used to generate the REV and the variance on the particle size in the REV. The sensitivity study was conducted for a case of $d=1.59 \mathrm{~mm}$ and porosity $\varepsilon=0.37$. Models were generated with 25,50 and 100 primitives with diameter variations of $\pm 10 \%, 20 \%$ and $40 \%$ of the nominal (mean) diameter $d$. Simulations are reported for an inlet air temperature of $25^{\circ} \mathrm{C}$ and a solid temperature of $250^{\circ} \mathrm{C}$ and with constant air properties at $25^{\circ} \mathrm{C}$. Fig. $2 \mathrm{~b}$ shows the portion of a generated REV that requires discretization for the CFD simulation. Pore-level simulations of the REVs were carried out using the commercial CFD software ANSYS ${ }^{\circledR}$ CFX $^{\mathrm{TM}}$ (ANSYS ANSYS CFX). Since the meshing size of the REV varies with the porosity and sphere diameter, the criteria of grid independence of the domain was based on the ratio of mean sphere diameter $(d)$ to the meshing element size.

Table 1 summarizes the grid-independence study conducted on a YADE-generated REV using 50 spherical primitives and $20 \%$ diameter variance for a flow $R_{d}=6.5$ and a temperature difference between the solid particles and the incoming air of $225^{\circ} \mathrm{C}$. The analysis shows that grid independence is achieved to within $6 \%$ for the normalized pressure and $1.1 \%$ for Nusselt number $\left(N u_{\mathrm{d}}\right)$ when the grid ratio reaches approximately 38 , which reflects a mesh size of the order of four million cells. All subsequent computations were produced with a similar grid ratio.

A study on the number of primitives required for sphericalvoid-phase porous materials was conducted by Dyck and Straatman (2015) and by Khan and Straatman (2016) for turbulent flow in domains containing large spherical objects. However, because of the apparent differences between the cases, the study was repeated in this work to ensure that the REVs were suitable. Computations were conducted for REVs containing 25, 50 and 100 spherical primitives with $20 \%$ diameter variance at $R_{d}=6.5$. Fig. 4 shows the results of the dimensionless heat transfer (in terms of the Nusselt number) as a function of the number of primitives and makes clear that there is little change in the predicted heat transfer beyond 50 primitives.

Fig. 5 shows the results for heat transfer for 50 primitives and particle variations of \pm 0 - $40 \%$ (of the mean diameter) for the same

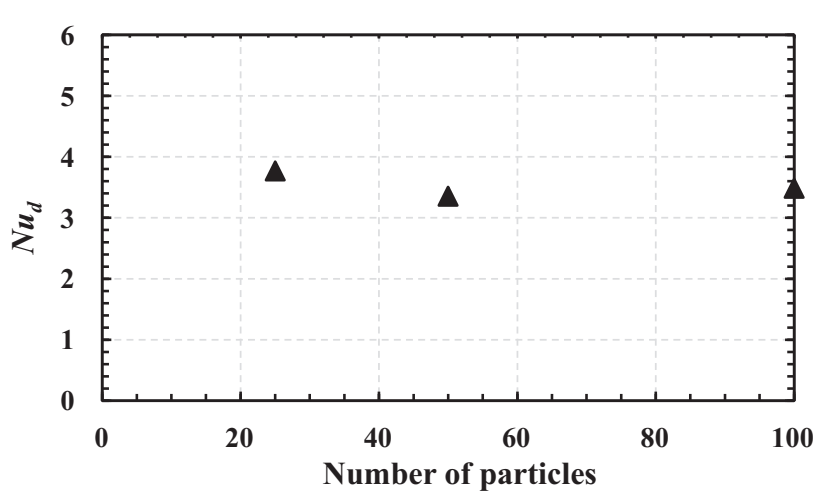

Fig. 4. Nusselt number as a function of the number of particles at fixed diameter of $d=1.59 \mathrm{~mm}$. Computations are for $R e_{d}=6.5$ and a temperature difference of $225^{\circ} \mathrm{C}$

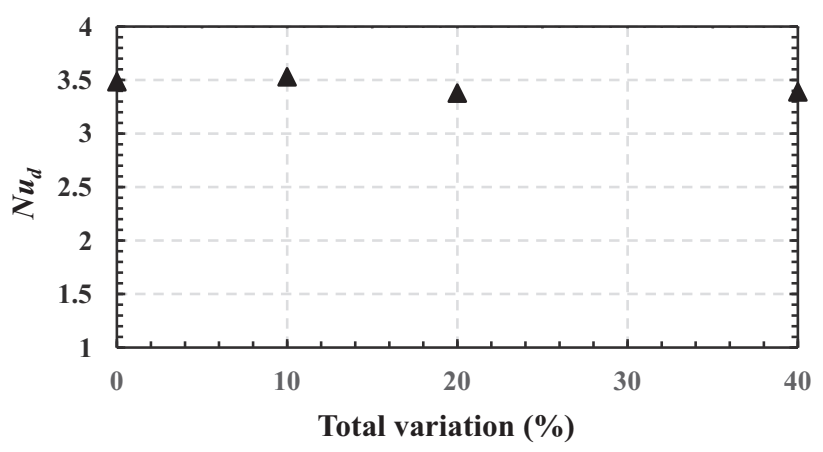

Fig. 5. Nusselt number as a function of particle diameter variation. Computations are for a Reynolds number $R e_{d}=6.5$ and a temperature difference of $225^{\circ} \mathrm{C}$.

thermofluid conditions considered in Fig. 4. This figure shows that even large particle size variations produce minimal changes in the predicted heat transfer. This is mainly because the interstitial surface area is only weakly influenced by the variation of sphere diameter; i.e. the surface area of the larger spheres compensates the surface area of the smaller ones. As a result, the total surface area with respect to the REV volume is nearly the same for a particular mean particle diameter, independent of the particle diameter variance, provided a large enough number of primitives is used to produce the geometric model.

Thus, on the basis of the computational data shown in Figs. 4 and 5 , we summarize that a minimum of 50 primitives is required to produce a domain that can be considered a REV; and that particle deviations do not need to be considered as they have very little effect on the computed results for heat transfer and pressure drop.

\section{Results and discussion}

On the basis of the sensitivity study on primitive number and variation, a geometric model was developed using YADE that has the same basic properties as the segmented image model present in Section 3.1 and shown in Fig. 1. In this manner, a geometric

Table 1

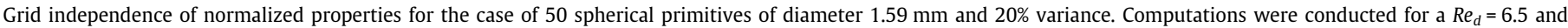
temperature difference of $225^{\circ} \mathrm{C}$.

\begin{tabular}{|c|c|c|c|c|c|}
\hline Cell number (million) & Particle diameter $(\mathrm{m})$ & Element size (m) & $\mathrm{d} / \mathrm{mesh}$ element & $\Delta P / L(\mathrm{~Pa} / \mathrm{m})$ & $\mathrm{Nu}$ \\
\hline 0.5 & $1.59 \times 10^{-3}$ & $1.40 \times 10^{-4}$ & 11.36 & 803.6 & 3.679 \\
\hline 1 & $1.59 \times 10^{-3}$ & $8.00 \times 10^{-5}$ & 19.88 & 947.5 & 3.554 \\
\hline 2 & $1.59 \times 10^{-3}$ & $5.50 \times 10^{-5}$ & 28.91 & 1038.6 & 3.476 \\
\hline 4 & $1.59 \times 10^{-3}$ & $4.20 \times 10^{-5}$ & 37.86 & 1110.4 & 3.429 \\
\hline
\end{tabular}


model was constructed with spherical particles of diameter $d=$ $0.510 \mathrm{~mm}$ and a target porosity of $\varepsilon=0.33$. To make the comparison volume similar to the segmented image model, the number of primitives was systematically adjusted until the edge length of the YADE model was close to the side length of the segmented image model. This required 512 primitives, which is well above the required number for the domain to be considered a REV based on the analysis presented in Section 3.3. The resulting idealized REV model is shown in Fig. 6. For clarity, Table 2 compares the geometric properties of the segmented image model with the idealized YADE model.

Computations were made on both the segmented image and YADE models for Reynolds numbers $\operatorname{Re}_{d} \in\{1,2.5,5,7.5,10\}$ and over a range of particle surface (wall) temperatures from 323 to $923 \mathrm{~K}$ with a fixed inlet air temperature of $298 \mathrm{~K}$. The effect of temperature dependence on air properties was included in all computations. The range of particle surface temperature was chosen to be consistent with temperatures observed in applications of smoldering combustion. Fig. 7 shows the results for pressure drop as a function of $R e_{d}$ for both geometric models. The figure indicates that while the trend of increasing pressure drop with $R e_{d}$ is similar, the magnitude of the pressure drop of the segmented-image REV is slightly higher, which we attribute mainly to the shape differences of the particles and the difference in internal area of the two REVs. The difference varies from $20 \%$ at very low $R e_{d}$ to approximately $6 \%$ at $R e_{d}=10$. Some modification to the particle shape in the YADE REV would result in increased interstitial area and would bring the results much closer together. This result is mainly of interest if it is a pressure difference that is specified to produce a given flow of air. If the mass flow rate of air is specified, the pressure drop is not as critical a parameter.

Fig. 8 gives a comparison of temperature fringe plots on a cut through the center $y-z$ plane of the segmented-image and YADE REVs to show the differences in the REV cross-sections and the similarity of the temperature contours in the domain. The image shows that even at $R e_{d}=10$, the air temperature rapidly reaches the particle surface temperature and nears thermal equilibrium over a very short length of the domain. This is the case for all of the high-temperature results, which lends credence to the approach taken to establish the convective heat transfer coefficient using the log-mean temperature difference, as shown in Eqs. (18) and (19). Fig. 9 gives the results of interstitial Nusselt number as a function of $R e_{d}$ for both geometric models. Here, the heat transfer results are shown to deviate by approximately 23\% at low $R e_{d}$, reducing to better than $4 \%$ at $R e_{d}=10$. Since the porosity is a near match between the geometric models, it is likely that the differ-

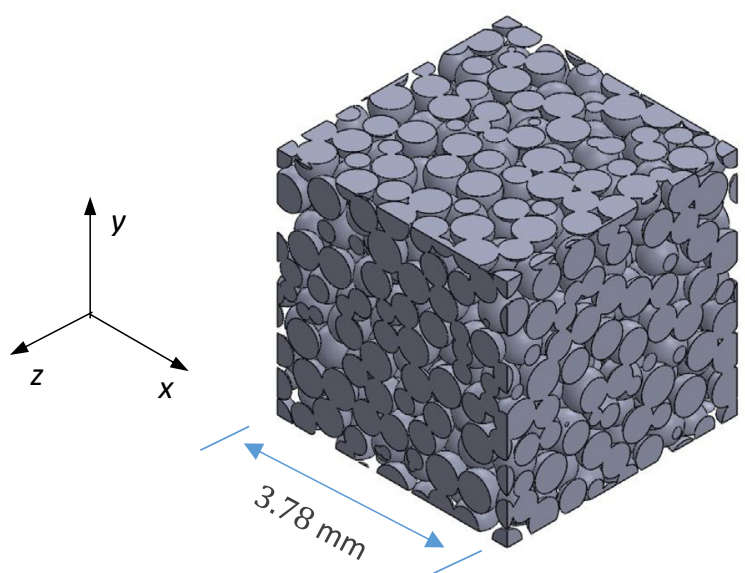

Fig. 6. YADE cubic REV of spherical particles with same geometric properties as the segmented image REV shown in Fig. 1.
Table 2

Comparison of geometric properties of the segmented image model produced from the data of Molnar (2015) with the idealized YADE model.

\begin{tabular}{llll}
\hline Model & Mean particle diameter $(\mathrm{mm})$ & Porosity $\varepsilon$ & $a_{s f}$ \\
\hline Segmented image & 0.510 & 0.33 & 8030.16 \\
YADE & 0.510 & 0.33 & 6522.35 \\
\hline
\end{tabular}

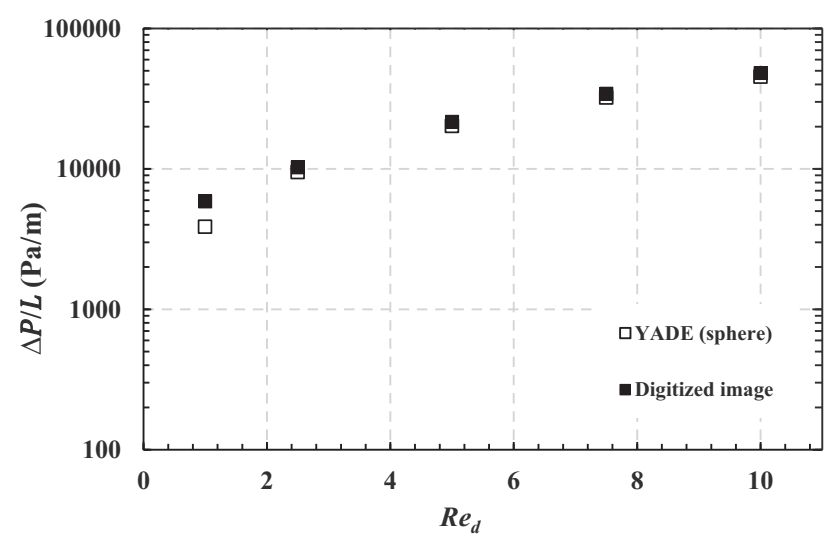

Fig. 7. Comparison of Pressure drop as a function of $R e_{d}$ through the segmented (Digitized) image and the mathematical YADE model.

ence in interstitial area (see Table 2) is the main cause of the heat transfer difference; although across the range of $R e_{d}$ considered, the convective heat transfer coefficients from the two models is considered to be sufficiently close. Considering the relative costs and times to generate the REV models, the YADE-REV has a clear advantage over the segmented-image model. Such a model can be generated in minutes and discretized and run over a range of parameters in hours, which makes studying the influences of geometric parameters extremely time-effective. As mentioned, some improvement could also be realized by making particle shape adjustments in YADE, although some study would be required on how to properly parameterize the particle shape.

The heat transfer results from the YADE-REV are compared with the experimental results of Wakao et al. (1979) and Zanoni et al. (2017) in Fig. 10. Note that the experimental results of Wakao et al. were derived from experiments on large spheres (10-130 $\mathrm{mm}$ ), while the results from Zanoni et al. (2017) were derived from a column of fine sand $(0.125-2 \mathrm{~mm})$. The plot given in Fig. 10 shows that the results derived from pore-level simulations on the YADE model are in reasonable accordance with the Wakao et al. (1979) model in terms of magnitude and trend, however, it is noted that the published results of the Wakao model are extrapolated to the range below $R e_{d}=15$. Compared to the experimental results of Zanoni et al. (2017), the present results give the same trend in terms of $R e_{d}$, but are considerably higher across the range of Reynold number. The large differences could be due to the reverse approach used in Zanoni et al. (2017) to obtain the results, which required an estimation of the heat loss from the test rig and an accurate estimate of the energy input over the heating period. It could also be partly attributed to the fact that computations were done herein for a heating condition, whereas the experimental results in Zanoni et al. (2017) were derived from a combination of heating and cooling. All things considered, the results of interstitial exchange produced using the YADE-REV are considered to be of reasonable accuracy relative to the highly-cited Wakao et al. (1979) model and, therefore, the approach is considered viable.

Additional processing is done to show the impact of solid temperature on the heat transfer between the particles and air. The heat transfer coefficient $h_{f s}$ is not a thermophysical property, but 


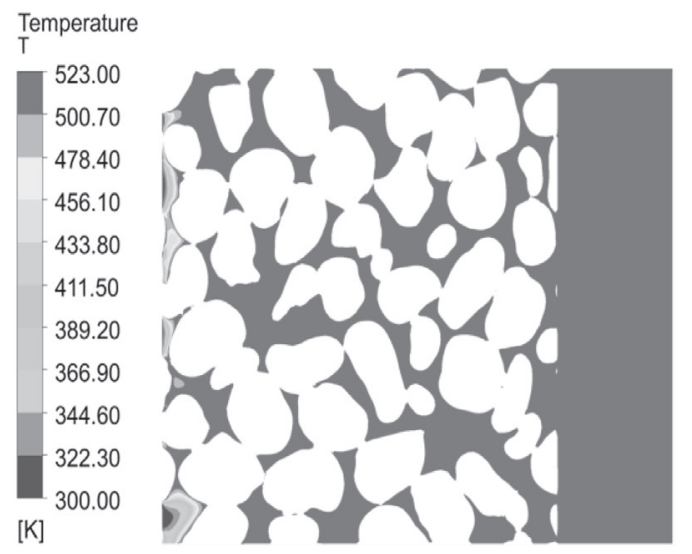

a)

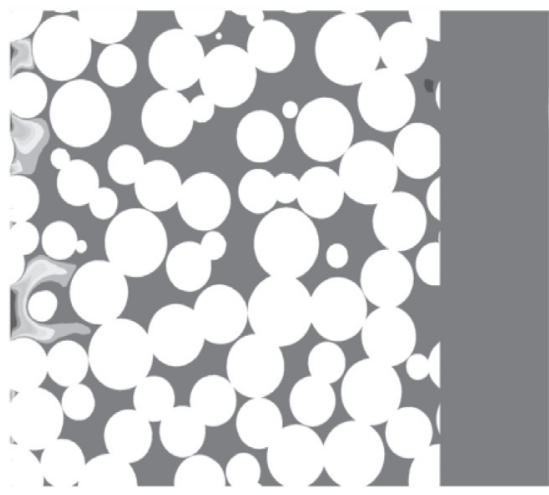

b)

Fig. 8. Mid-plane $(x-y)$ distribution of fluid temperature variations for (a) Segmented image REV and (b) YADE REV. For these cases, $R e_{d}=10$ and $T_{s}=523 \mathrm{~K}$.

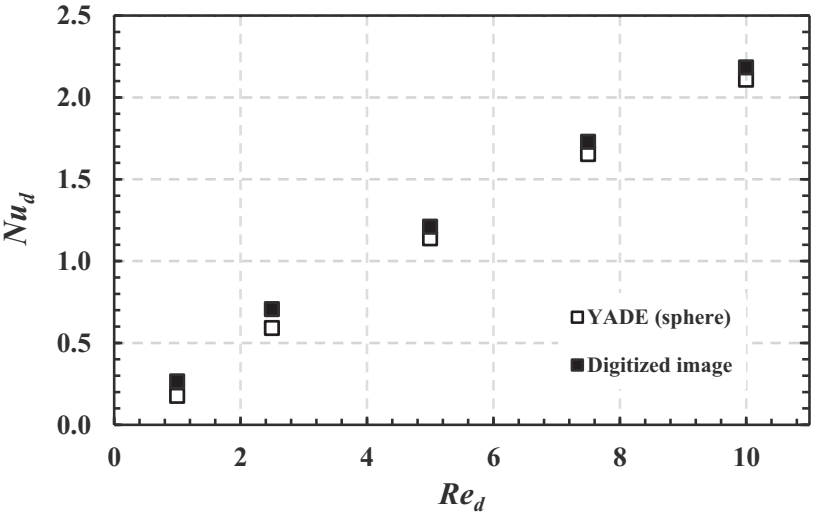

Fig. 9. Comparison of Nusselt number as a function of Reynolds number for the segmented (Digitized) image and the mathematical YADE model at a particle surface to inlet temperature difference of $25^{\circ} \mathrm{C}$.

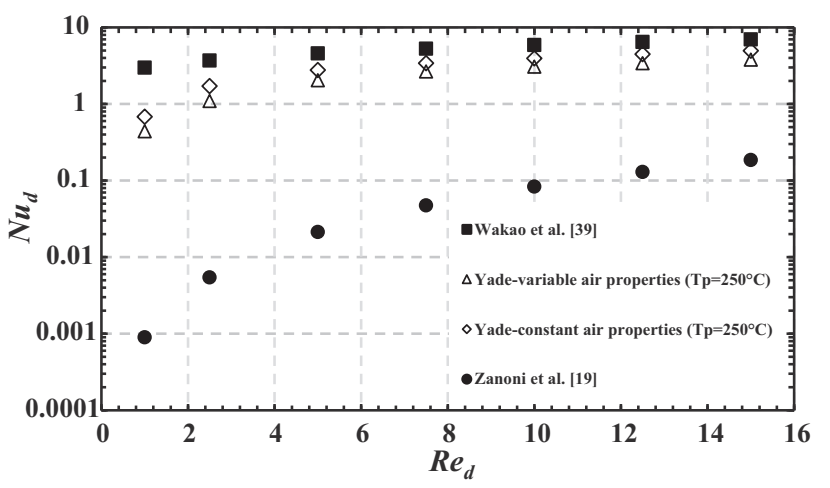

Fig. 10. Nusselt number as a function of Reynolds number for YADE cases compared to the experimental data of Wakao et al. (1979) and Zanoni et al. (2017). Note that the data of Wakao et al. (1979) is extrapolated backward below $\operatorname{Re}_{d}=15$.

rather an interface property that is dependent on thermophysical properties like viscosity, conductivity, flow regime and geometry. In this respect, if the temperature (bulk or surface) of the fluid varies in the flow direction, the value of the convective heat transfer coefficient (which depends on temperature-dependent properties of the fluid) can also vary. Because of the high temperature deviations prevalent in applications like smoldering combustion, the influence of the temperature on air properties is considered important.

The variation of pressure drop $\Delta P / L$ with the Reynolds number and particle temperatures is shown in Fig. 11. The plot shows that pressure drop increases with increasing $R e_{d}$, and that the pressure drop at a particular $R e_{d}$ decreases with increasing particle temperature. The hydraulic behavior can also be characterized in terms of the permeability, which for weak flows is usually approximated from the Karman-Kozeny expression (Vafai, 2015; Kaviany, 1995):

$K=\frac{\varepsilon^{3} d^{2}}{a(1-\varepsilon)^{2}}$

which gives a value $K=2.263 \times 10^{-09} \mathrm{~m}^{2}$ for the case of $d=1.59$ $\mathrm{mm}$ and $\varepsilon=0.37$. The result computed from the pore-level simulation is $K=1.79 \times 10^{-09} \mathrm{~m}^{2}$, which is of similar order and magnitude. The difference may be due to the fact that most of the computed results fall outside the region of creeping flow for which the Karman-Kozeny expression is valid.

Fig. 12 shows that accounting for the influence of temperature on air properties also has a significant impact on the heat transfer results. In this case, raising the particle temperature has the expected net effect of reducing the local Nusselt number, while the trend over the range of $R e_{d}$ considered remains the same. Fig. 13 shows the same data presented in Fig. 12, but this time as a function of temperature difference $T_{s}-T_{\text {in }}$ for the different $R e_{d}$ considered. This plot makes clear how important it is to consider

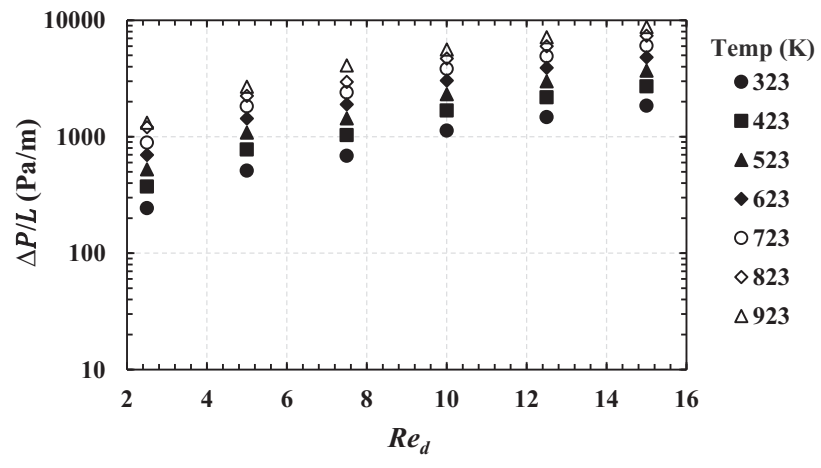

Fig. 11. Pressure drop as a function of $R e_{d}$ for YADE model over a range of particle surface temperature. The air inlet temperature is fixed at $298 \mathrm{~K}$. 


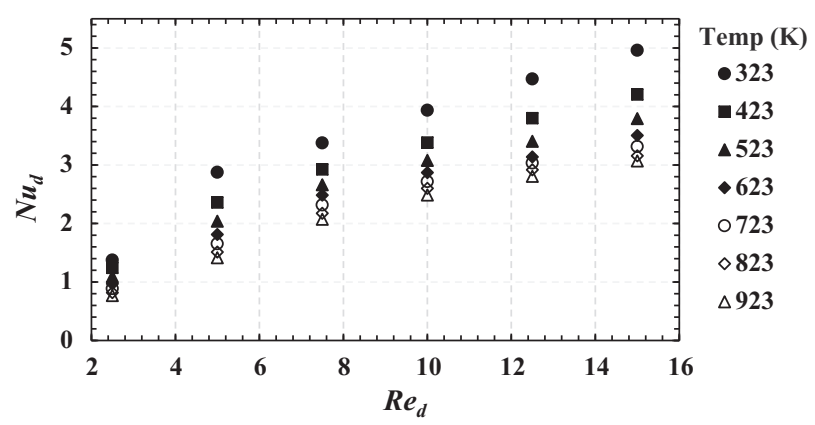

Fig. 12. Nusselt number as a function of $R e_{d}$ for YADE model over a range of particle surface temperature. The air inlet temperature is fixed at $298 \mathrm{~K}$.

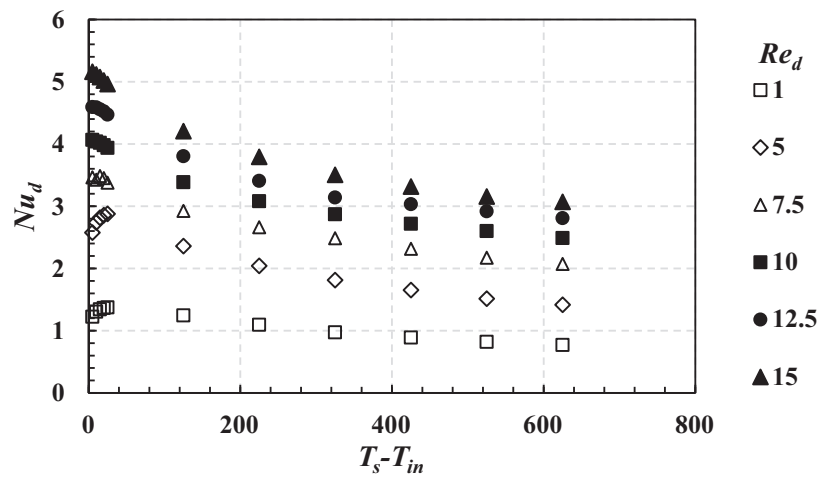

Fig. 13. Plot showing the Nusselt number as a function of temperature difference for several values of $\mathrm{Re}_{d}$.

property variations with $T$ even for moderate temperature differences. In the range of $5<\Delta T<50^{\circ} \mathrm{C}$, the heat transfer is seen to vary $10-15 \%$ in some cases followed by a monotonic decrease with increasing temperature. Based on the results shown in Figs. 12 and 13 , a correlation for the Nusselt number is proposed that takes into account the temperature effect on thermofluid properties. This is not intended to be a considered a universal correlation, rather it is presented as a compact means of providing a correlation for porous-continuum computations that consider local thermal non-equilibrium. Because detailed information on the thermofluid properties and the temperatures of the constituents are available from the present calculations, a correlation similar to Kalganova's (Young and Pfender, 1987) is adopted:

$N u_{d}=\mathrm{a} \frac{k_{w}}{k_{\infty}}+\mathrm{b} \operatorname{Pr}_{\infty}^{1 / 3} \operatorname{Re}_{\infty}^{0.5}\left(\frac{\rho_{\infty} \mu_{\infty}}{\rho_{w} \mu_{w}}\right)^{c}\left(\frac{c_{p \infty}}{c_{p w}}\right)^{d} \operatorname{Pr}=0.7$

where the variables are as follows: $k_{w}, \rho_{w}, \mu_{w}$ and $c_{p w}$ are the conductivity, density, dynamic viscosity and specific heat of the fluid at the particle surface temperature; $k_{\infty}, \rho_{\infty}, \mu_{\infty}$ and $c_{p \infty}$ are the same properties at the inlet ambient temperature; and $a, b, c$ and $d$ are coefficients that must be established based on a fit to experimental or computational data. Using the regression analysis package available in the Matlab ${ }^{\mathrm{TM}}$ software, the values shown in Table 3 are pro-

Table 3

Summary of coefficients for Eq. (21) for the YADE geometric model with $d=1.59 \times 10^{-03} \mathrm{~m}$.

\begin{tabular}{ll}
\hline $\mathrm{a}$ & -0.376 \\
$\mathrm{~b}$ & 1.579 \\
$\mathrm{c}$ & -2.077 \\
$\mathrm{~d}$ & -3.789 \\
\hline
\end{tabular}

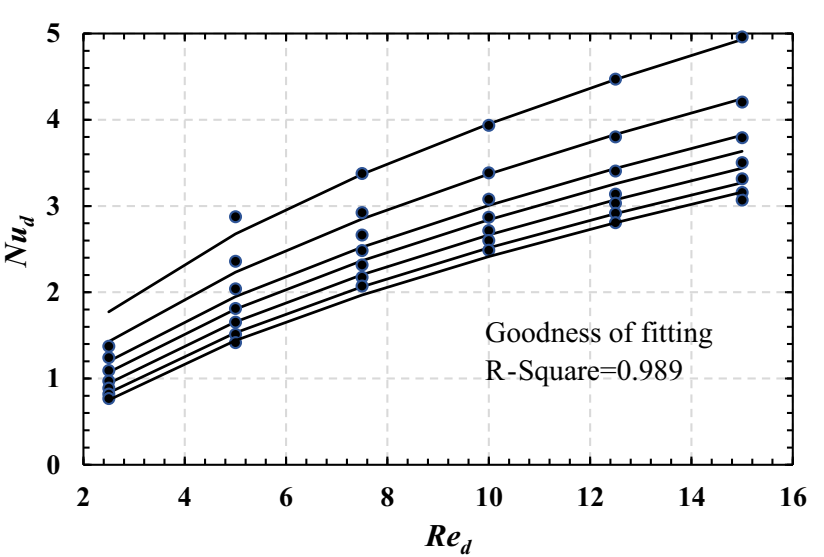

Fig. 14. Comparison between the simulations shown in Fig. 12 and the correlation given in Eq. (21) with the coefficients in Table 3. For clarity, all data are all shown as black dots and the curves are all shown as solid lines with the lower line corresponding to $T_{s}=923 \mathrm{~K}$ and the upper line corresponding to $T_{s}=323 \mathrm{~K}$. The quality of the correlation is 0.989 , which is excellent.

duced, yielding a close fit to the data for the given REV. Fig. 14 shows all of the data from CFD simulations as points, and curves of $N u_{d}$ from Eq. (21) using the coefficients in Table 3. What is interesting is that the leading coefficient is negative, which implies that for $R e_{d} \rightarrow 0$, the interstitial exchange becomes negative, which is not sensible. Clearly, the expression is only valid over the range $0.1 \leq R e_{d} \leq 15$, which is the range over which simulations were conducted. The purpose of the correlation is not to propose a universal heat transfer correlation for a packed bed of spherical particles, but rather to show that heat transfer data from a parametric study on a mathematically generated REV can provide a useful and compact expression to compute the local heat transfer coefficient in computational models that utilize the volume-averaged approach under local thermal non-equilibrium conditions. While the proposed expression is valid only for the geometric case considered, the YADE approach makes it easy to develop similar expressions for any REV under consideration.

\section{Summary}

A study has been presented that compares a geometric model derived from segmented images of packed sand to a mathematical YADE model over a range of flow and heating conditions that are relevant to smoldering combustion. Comparisons of pressure drop and convective heat transfer at low Reynolds numbers shows that the YADE model gives results that are similar to the segmented image model and close to other documented results for packed beds of particles. In this respect, the mathematical YADE model is considered a viable approach for studying pore-level transport. The main advantage of the YADE approach is that a representative elemental volume (REV) of packed particles can be generated for a specific particle diameter and target porosity in minutes and then discretized and simulated in hours to produce parameters that can be used in volume-averaged calculations. Such a geometric model was used herein to study hydrodynamics and convective heat transfer, but it can also be used to study mass transfer and chemical reaction associated with combustion. A correlation was proposed to describe the Nusselt number over a wide range of temperatures at low- $R e_{d}$. Such a correlation is useful for conducting heat transfer studies on much larger domains using a non-equilibrium porous continuum approach. 


\section{Acknowledgements}

The authors gratefully acknowledge the financial support from the Natural Engineering and Sciences Research Council of Canada and the Ontario Graduate Scholarship (Thabet).

\section{References}

ANSYS. ANSYS CFX, vol.16.0.

ANSYS CFX-Pre User's Guide. Release 16.0.

ANSYS. Fluent, vol.16.0.

ANSYS Fluent Theory Guide. Release 16.0.

ANSYS ICEM CFD User's Manual, Release 16.0.

ANSYS, Workbench Meshing Tool, vol.16.0.

Buchgraber, M., Al-Dossary, M., Ross, C.M., Kovscek, A.R., 2012. Creation of a dualporosity micromodel for pore-level visualization of multiphase flow. J. Pet. Sci. Eng. 86, 27-38.

Chueh, C.C., Bertei, A., Pharoah, J.G., Nicolella, C., 2014. Effective conductivity in random porous media with convex and non-convex porosity. Int. J. Heat Mass Transf. 71, 183-188.

Cundall, P.A., Strack, O.D., 1979. A discrete numerical model for granular assemblies. Geotechnique 29 (1), 47-65.

Dassault Systemes, Solidworks Educational Version 2015-2016.

Dyck, N.J., Straatman, A.G., 2015. A new approach to digital generation of spherical void phase porous media microstructures. Int. J. Heat Mass Transf. 81, 470-477.

Kar, K., Dybbs, A., 1982. Internal heat transfer coefficient of porous metals. NASA STI/Recon Tech. Rep. A 84, 81-91.

Karimian, S.M., Straatman, A.G., 2008. CFD study of the hydraulic and thermal behavior of spherical-void-phase porous materials. Int. J. Heat Fluid Flow 29 (1), 292-305.

Kaviany, M., 1995. Principles of Heat Transfer in Porous Media. Springer, New York, NY

Khan, F.A., Fischer, C., Straatman, A.G., 2015. Numerical model for non-equilibrium heat and mass exchange in conjugate fluid/solid/porous domains with application to evaporative cooling and drying. Int. J. Heat Mass Transf. 80, $513-528$.

Khan, F.A., Straatman, A.G., 2016. Closure of a macroscopic turbulence and nonequilibrium turbulent heat and mass transfer model for a porous media comprised of randomly packed spheres. Int. J. Heat Mass Transf. 101, 1003-1015.

Molnar, I.L., Gerhard, J.I., Willson, C.S., O'Carroll, D.M., 2015. The impact of immobile zones on the transport and retention of nanoparticles in porous media. Water Resour. Res. 51 (11), 8973-8994.
Molnar, I.L., 2015. Silver Nanoparticle Transport Through Soil: Illuminating the Governing Pore-Scale Processes. PhD Thesis, Western University. Electronic Thesis and Dissertation Repository. 3394. <http://ir.lib.uwo.ca/etd/3394>.

Nakayama, A., Ando, K., Yang, C., Sano, Y., Kuwahara, F., Liu, J., 2009. A study on interstitial heat transfer in consolidated and unconsolidated porous media. Heat Mass Transf. 45 (11), 1365-1372.

Nield, D.A., Bejan, A., 2017. Convection in Porous Media. Springer, New York.

Natural Resources Canada [Online]. Available: <http://www.nrcan.gc. ca/forests/fire-\%0Ainsects-disturbances/fire/13143\%0A> (accessed: 10-May2017).

Rein, G., 2009. Smouldering combustion phenomena in science and technology. Int Rev. Chem. Eng. 1, 3-18.

Rivers, M.L., Citron, D.T., Wang, Y., 2010. Recent developments in computed tomography at GSECARS. In: Proc. SPIE, Dev. X-Ray Tomogr. VII, vol. 7804, p. 780409.

Savron Smoldering Solutions [Online]. Available: <http://www.savronsolutions. com> (accessed: 30-May-2017).

Šmilauer, V., Chareyre, B., 2011. Yade Documentation.

Soulaine, C. Direct Numerical Simulation in Fully Saturated Porous Media [Online] Available: <https://web.stanford.edu/ csoulain/PORE_SCALE/Chap2.pdf>.

Vafai, K., 2015, Handbook of Porous Media, Boca Raton.

Wakao, N., Kaguei, S., Funazkri, T., 1979. Effect of fluid dispersion coefficients on particle-to-fluid heat transfer coefficients in packed beds: correlation of Nusselt numbers. Chem. Eng. Sci 34 (3), 325-336.

Wakao, N., Kaguei, S., 1982. Heat and MassTransferinPackedBeds. McGraw-Hill, NewYork.

Whitaker, S., 1997. Volume Averaging of Transport Equations. Fluid Transport in Porous Media, Springer, Southampton, UK, pp. 1-59.

Yang, J., Wang, Q., Zeng, M., Nakayama, A., 2010. Computational study of forced convective heat transfer in structured packed beds with spherical or ellipsoida particles. Chem. Eng. Sci. 65 (2), 726-738.

Yang, J., Wang, J., Bu, S., Zeng, M., Wang, Q., Nakayama, A., 2012. Experimental analysis of forced convective heat transfer in novel structured packed beds of particles. Chem. Eng. Sci. 71, 126-137.

Young, R.M., Pfender, E., 1987. Nusselt number correlations for heat transfer to small spheres in thermal plasma flows. Plasma Chem. Plasma Process. 7 (2), 211-229.

Yu, Q., Thompson, B.E., Straatman, A.G., 2006. A unit cube-based model for heat transfer and fluid flow in porous carbon foam. J. Heat Transf. 128 (4) 352-360.

Zanoni, M.A.B., Torero, J.L., Gerhard, J.I., 2017. Determination of the interfacial heat transfer coefficient between forced air and sand at reynold's numbers relevant to smouldering combustion. Int. J. Heat Mass Transf. 114, 90-104. 